\title{
BPMSS - Lonza's Biopharmaceutical Small Scale Plant Started cGMP Manufacturing in September 2004
}

\author{
Frans W.J.M.M. Hoeks, Diego R. Schmidhalter*, Rainer Gloeckler, Christoph Herwig, \\ Karen Theriault, Sven Frie, Wouter van den Broek, and Frank Laukel
}

\begin{abstract}
In September 2004 Lonza (Visp, Switzerland) started operations in a new state of the art facility for cGMP manufacture of injectable grade microbially derived biopharmaceuticals. Both intracellular and secreted products can be processed in the new production line, which delivers bulk drug substances. cGMP conformity, including clean room classification, was cross-checked during the design phase with the Swiss regulatory authorities. The layout of the highly automated multi-product line is based on a high performance $1000 \mathrm{I}$ bioreactor suitable for high cell density fermentation. Besides standard chromatography and ultrafiltration, the ex-proof design in upstream and downstream areas allows for solvent feeds in fermentation and for implementation of HPLC purification steps in protein and peptide purification.
\end{abstract}

Keywords: cGMP · Chromatography · Custom manufacturing · Ex-proof · Intracellular and extracellular · Lonza · Microbial biopharmaceuticals

\section{Introduction}

Lonza Custom Manufacturing offers the life science industry world-class services related to research, development, and manufacturing of chemicals, intermediates, active pharmaceutical ingredients (API's) and biologics from clinical to commercial quantities. As the leading custom manufacturer, Lonza is able to run more than one hundred development and manufacturing programs in parallel at any time. Lonza runs high cell density microbial fermentation up to 75,000 1 scale at Kounim in the Czech Republic. Large-scale protein purification is established at Lonza's mammalian cell culture facility in Portsmouth (NH, USA) with up to $2 \mathrm{~m}$ column diameter for purification of 20,000 1 batches. In May 2002, the board of directors of Lonza Ltd. took the decision to build new state-of-the-art plants for injectable grade microbially derived biopharmaceuticals.

\footnotetext{
${ }^{\star}$ Correspondence: Dr. D.R. Schmidhalter Head of Microbial R\&D LBP

Lonza AG

CH-3930 Visp

Tel.: +4127948 6252

Fax: +412794762 52

E-Mail: diego.schmidhalter@lonza.com
}

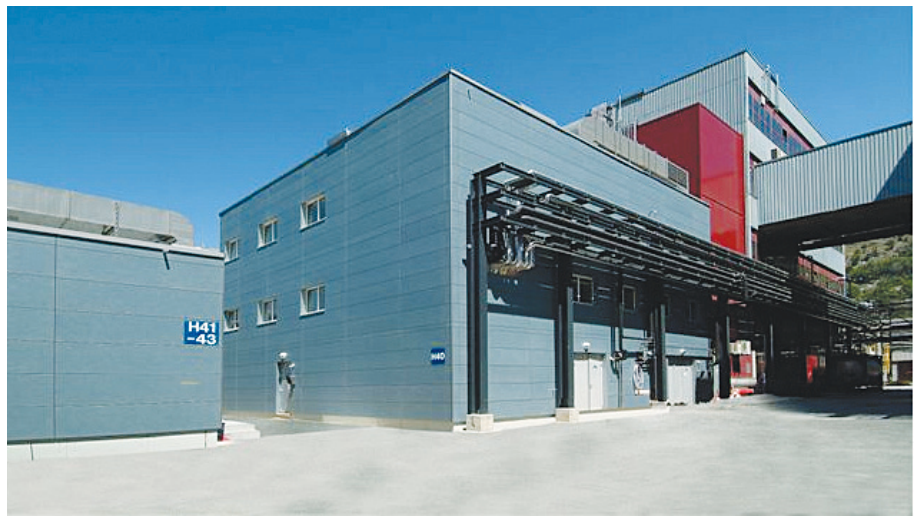

The expansion into microbially derived biopharmaceuticals comprised a 10001 fermenter, which was called BPMSS, an acronym for biopharmaceutical manufacturing small scale. Furthermore, setting up a 15,000 1 line is envisaged. The 10001 facility (Fig. 1) has been built in Visp adjacent to the existing launch plant for Lonza's traditional microbial business, which already provides small molecules and secondary metabolites to the pharmaceutical industry. By building the biopharmaceutical facility in Visp, synergies with the very experienced microbial research and development group and with the large predominantly chemical operation with a sizable skilled workforce of approximately 2500 employees could be fully exploited. Strain design, process development and manufacturing, plus an analytical and regulatory package are offered to Lonza's customers on an exclusive basis. Biopharmaceutical products for clinical and commercial use are being manufactured in the 10001 facility. These products are in general purified bulk solutions of recombinant proteins and large peptides, as well as DNA. Examples of products are hormones, fragment anti-bodies and protein vaccines typically produced by microorganisms such as Escherichia coli, Pichia pastoris, and Saccharomyces cerevisiae. In this paper the versatile technical capabilities of the 10001 fermentation plant, including purification, will be described by using an example. 


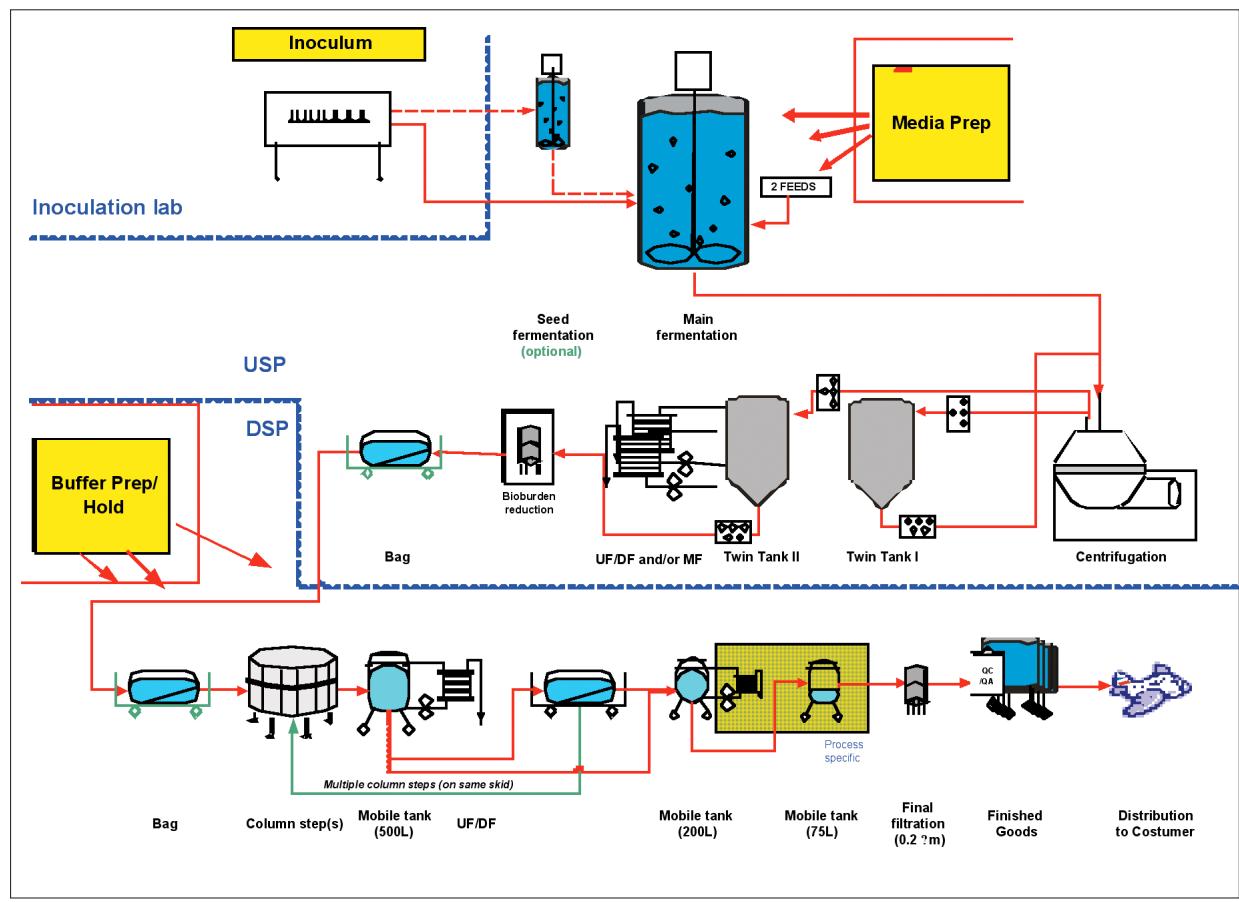

Fig. 2. Scheme of the $1000 \mathrm{I}$ line for the production of biopharmaceuticals

\section{Facility Overview}

BPMSS was designed for flexible processing and can accommodate a large variety of unit-operations, e.g. fermentation, separation, micro-filtration, homogenisation, refolding, ultra-filtration, chromatography, HPLC, bulk filtration, bulk fill. An example of a sequence of unit-operations of a process is given in Fig. 2. The facility has two completely separated and dedicated areas, one for upstream processing, i.e. fermentation, harvesting and refolding and one for purification. The whole plant is ex-proof.
So, solvents can be used both in fermentation and in purification. The upstream area has a laboratory area for the preparation of inoculum for the fermentation and a clean room for all further processing before purification. The purification area has separate clean rooms for buffer solution preparation, ultra-filtration, chromatography and bulk filling. Clean room design requirements are adapted for different production areas. The facility is divided into processing areas, where open and closed production occurs, and general support areas, where goods and materials are handled and transported. The

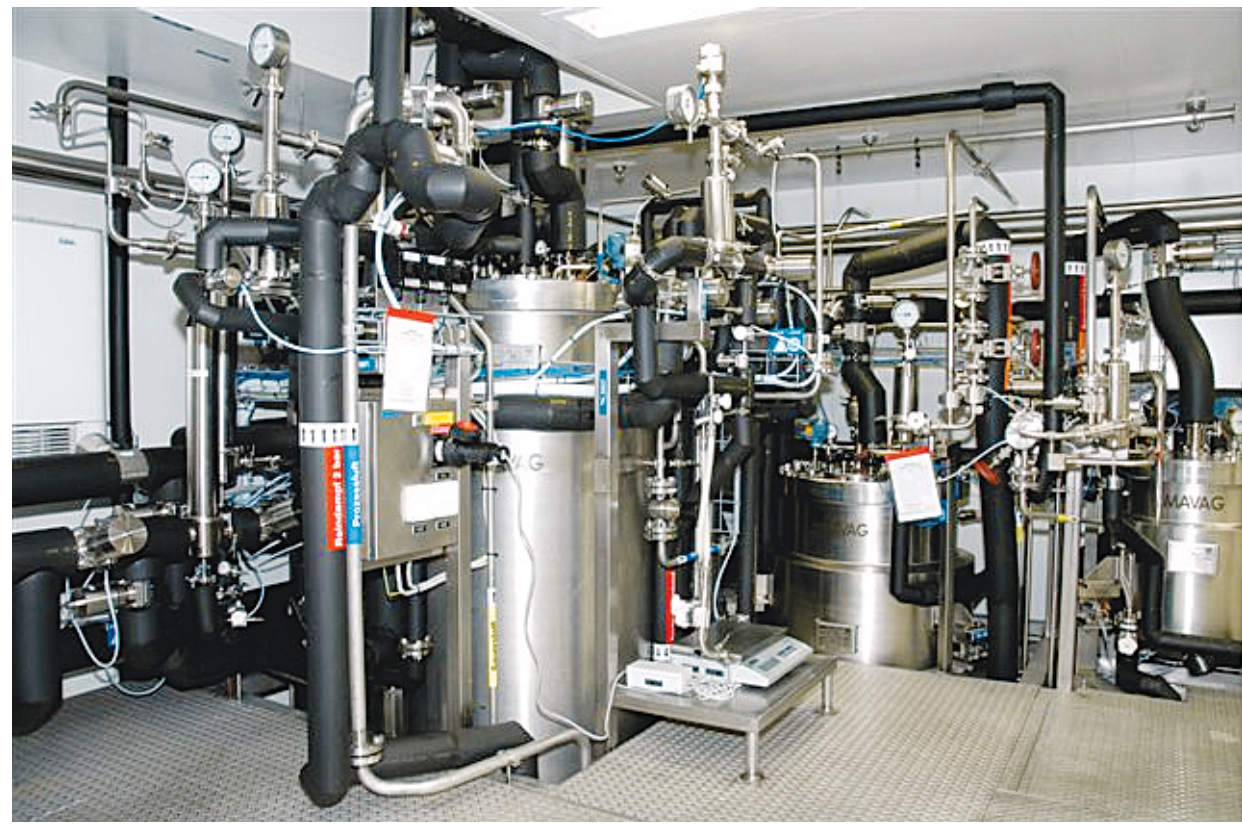

Fig. 3. 1000 I High-performance bioreactor for the production of biopharmaceuticals areas are separated by airlocks and gowning areas.

The plans for the cGMP conformity of the BPMSS facility, including the clean room classification from $\mathrm{CNC}$ to class $\mathrm{C}$ were approved by the relevant Swiss authority (Swissmedic) during the design phase.

\section{Process Description Fermentation}

The growth of microbial cells is initiated by inoculating growth medium in a shakeflask with a few millilitres of cell suspension which has been stored at $-80^{\circ} \mathrm{C}$. The shakeflask is then incubated in a shaker under controlled environmental conditions in order to provide enough oxygen and mixing to the growing micro organisms. This process step is run in a cleanroom environment, which is also suitable for master cell banking.

After cultivation, the shakeflask is aseptically connected to the 10001 production fermenter, which contains sterile growth medium. The conditions promote fast growth and product formation in batch or fed-batch mode. Intensive stirring, a high aeration rate and in some cases pure oxygen are used to obtain high cell densities in order to maximise the amount of product. Further additions of nutrients are possible to maintain optimal conditions. The growth of the micro-organism may be controlled by feeding, e.g. glucose or methanol to the fermenter. Product formation based on the recombinant genetic information in the micro-organism may be triggered by addition of an inducer, e.g. lactose or methanol or by applying a specific temperature profile. The protein product will be 'expressed' i.e. formed after induction, resulting in a product concentration of up to $4 \mathrm{~g} / \mathrm{l}$.

The 1,000 litre production fermenter (Fig. 3) is a stirred tank reactor capable of operating at a final working volume of up to 8001 (950 1 with gas hold-up). State-ofthe-art features are the duplicated temperature, dissolved oxygen and $\mathrm{pH}$ probes and exhaust gas analysis. Spare ports for additional measurements are provided in order to accommodate further specific analyses e.g. FIA, on-line HPLC.

Oxygen-enriched air can be provided to the sparger to maintain adequate dissolved oxygen at high mass transfer rates of up to $400 \mathrm{mmol} \mathrm{O}_{2} / \mathrm{kg}$.h to ensure high cell density cultivation. This is considerably higher than the industry standard of 100-200 mmol $\mathrm{O}_{2} / \mathrm{kg}$.h. The cooling capacity is designed accordingly to remove the heat produced during the bioprocess.

\section{Process Description Primary Recovery}

A continuous disk stack centrifuge is used to separate cells from the culture su- 
pernatant after fermentation. A microfiltration unit is used for final cell removal or may replace the centrifugation step altogether. Homogenisation up to 1500 bar can be carried out to obtain intracellular product from microbial cells after harvest.

Two harvest tanks are installed, each with a working volume of 20001 . Product can be stored at $<8{ }^{\circ} \mathrm{C}$ prior to transfer to disposable containers.

Processing panels are provided with the harvest tanks for flexibility in operations to connect to any of the primary recovery equipment or bags.

After final cell removal from the product solution, concentration and conditioning by ultra-filtration/dia-filtration (UF/DF) can be carried out. The product is retained by the UF membranes, which enables a change of the medium or 'buffer'. The UF/DF skid is designed to process high volumes in less than $12 \mathrm{~h}$. Dia-filtration buffer is provided directly from bags and is also used to flush through the pipes to prevent product loss.

The plant is also suitable for the isolation, recovery and purification of inclusion bodies including the solubilisation and refolding of target proteins. The harvest tanks are suitable for handling guanidin- $\mathrm{HCl}$ and other aggressive solutions.

\section{Process Description Downstream Processing (DSP)}

After fermentation and primary recovery, the product is purified by a number of chromatography steps. Buffer exchange is carried out by UF/DF. The purification train is designed to handle approx. $2.6 \mathrm{~kg}$ of product at the start of purification, with subsequent unit-operations of $90 \%$ step efficiency.

A chromatography skid with three columns (450 and $630 \mathrm{~mm}$ diameter) and gel filtration columns of up to $500 \mathrm{~mm}$ height as well as a UF/DF skid are available. Preparative HPLC is carried out in the ex-proof environment (Fig. 4).

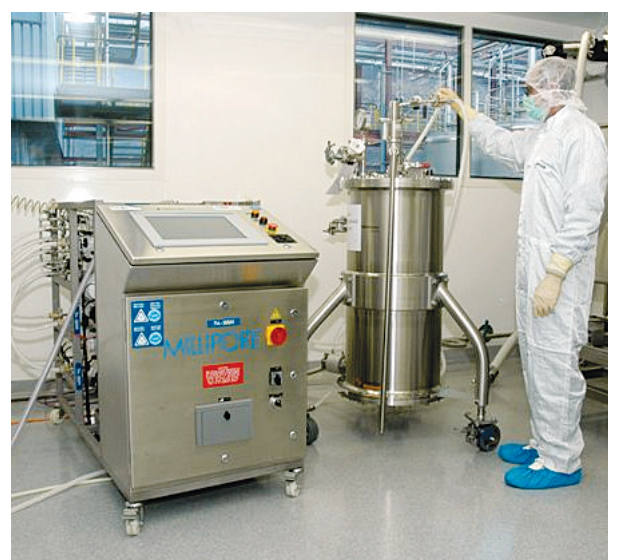

Fig. 4. Purification of injectable grade biopharmaceuticals in a cleanroom environment
Process steps can be operated at ambient or low $\left(7-15^{\circ} \mathrm{C}\right)$ temperatures. The temperature adjustment is done via buffer temperature control.

Buffers can be used directly or can be prepared in a concentrated form for in-line dilution.

The chromatography skids have in-line mixing capability for concentrated buffers and for gradient elution. Multiple column cycles may be used within a batch. All chromatography columns are packed semi-automatically using a mobile packing skid to ensure consistent and efficient packing of product-dedicated resins.

After purification the product is filtered through a bioburden reduction filter and aliquoted into sterile tanks or bags under laminar flow. Freezing of the product can also be carried out at $-80^{\circ} \mathrm{C}$.

For optimum flexibility, utility panels are provided in the downstream production rooms. Process liquids are handled in bags and mobile tanks, which enhances the flexibility and versatility to a very high level.

\section{Process Overview Production Support}

Solid components and liquids for fermentation media are handled in a separate media preparation area. Media are sterilized in the fermenter and in the feed tanks after transfer. Feeds that can not be heat sterilized are sterile-filtered before use.

Buffer preparation is carried out in a central buffer preparation area. The dispensed solid and liquid components are added to water in two mix tanks (600 and 1200 1). Buffers can be prepared with either purified water or water for injection. All buffers are transferred into sterile bags which are equipped with $0.2 \mu \mathrm{m}$ liquid inlet filters.

Both the fermentation and the purification areas have dedicated rooms for glass cleaning and autoclaving of materials.

\section{Process Description Cleaning in Place (CIP) and Steaming in Place (SIP)}

CIP is critical to the successful operation of the plant. The objective of the CIP process is to clean the equipment reproducibly (tanks and pipe-work) in situ using validated cleaning procedures. One CIP skid is dedicated to the cleaning of the upstream process equipment and one is dedicated to downstream equipment. All tanks and transfer lines are CIP-able. The fermenter and all equipment and transfer lines that can feed the fermenter are steam-sterilised in place. All other primary recovery and downstream equipment and transfer lines are steam-sanitized in place or, if equipment is not designed for steaming, chemically sanitised.
All CIP and SIP procedures are fully automated and recipe driven from the central control system. This creates a high degree of efficiency within the plant.

\section{Process Description Automation}

There is a single, plant-wide process control system (distributed control system; DCS), with integrated skid-based process control systems. Upstream operations are completely automated and recipe controlled. For quick adaptation of the recipe to a specific process, equipment modules, e.g. groups of valves, were defined. This allows to set entire transfer routes with a few options, hence programming of individual valves is avoided.

All DCS hardware is connected to an uninterruptible power supply and is 21CFR Part 11 compliant. All DCS software is configured according to a defined, documented, quality-controlled and Lonza-approved design. The system is required to support the process operation in an efficient, reliable and robust manner. System functionality allows for maximum possible configuration at the recipe level.

\section{Process Overview Utilities and HVAC}

USP purified water from the distribution system of the Visp site is used to prepare water for injection and clean steam. Process air, nitrogen and cooling and heating media are also obtained from the site. Two new HVAC systems are included in BPMSS facility. One HVAC system serves upstream processing and one serves the downstream processing area. Both systems provide $100 \%$ outside air with a once-through flow design because of the ex-proofing, and serve a range of clean room classes from $\mathrm{CNC}$ to Grade $\mathrm{C}$.

\section{Concluding Remarks}

A versatile 10001 fermentation and purification train was put into cGMP operation in September 2004 after approval by Swissmedic. Biopharmaceutical products have been produced since then on an exclusive basis for Lonza's customers.

Received: December 6, 2004 